\section{Unconditional and Conditional Quantitative Trait Loci Mapping for Plant Height in Nonheading Chinese Cabbage}

\author{
Yan Cheng', Qian Wang, and Qingyu Ban \\ State Key Laboratory of Crop Genetics and Germplasm Enhancement, \\ Nanjing Agricultural University, Nanjing 210095, China
}

Jianfeng Geng ${ }^{1}$ and Xiao Wei Zhang

Institute of Horticulture, Henan Academy of Agricultural Science, Zhengzhou, 450002, China

\begin{abstract}
Ying Yi and Xilin $\mathrm{Hou}^{2}$
State Key Laboratory of Crop Genetics and Germplasm Enhancement, Nanjing Agricultural University, Horticultural College, Weigang No. 1, Nanjing, Jiangsu 210095, China; and Key Laboratory of Southern Vegetable Crop Genetic Improvement, Ministry of Agriculture, Nanjing 210095, China
\end{abstract}

Additional index words. nonheading Chinese cabbage, plant height, unconditional QTL mapping, conditional QTL mapping

\begin{abstract}
Quantitative trait loci (QTLs) identified so far in Brassica were mainly generated in the final stage of plant development, which did not apply to the exploitation of genetic effects that were expressed during a specific developmental stage. Thus, the objective of this study was to simultaneously identify unconditional and conditional QTL associated with plant height at various stages of nonheading Chinese cabbage. One hundred twelve doubled haploid (DH) lines developed from the cross between nonheading Chinese cabbage lines 'SW-13' and 'SU-124' were used for QTL analysis of plant height by the composite interval mapping method combined with mixed genetic model. The map we used for QTL analysis was an updated version of the first genetic map of nonheading Chinese cabbage with 48 additional markers to the same DH population. With data from 2 years, a total of 11 unconditional QTLs in six linkage groups and 23 conditional QTLs in eight linkage groups were identified for plant height. The results indicated that the number and type of QTLs and their genetic effects for plant height were different in a series of measuring stages. Each QTL can explain $7.92 \%$ to $28.25 \%$ of the total phenotypic variation. Two QTLs (ph8-4 and ph8-5) were identified to be associated with plant height using both unconditional and conditional mapping methods simultaneously in 2 years. These results demonstrated that it is highly effective for mapping QTL of developmental traits using the unconditional and conditional analysis methodology.
\end{abstract}

Brassicas includes many important vegetables such as Chinese cabbage [Brassica rapa subsp. Pekinensis (Lour.) Hanelt], nonheading Chinese cabbage [B. rapa subsp. Chinensis (L.) Hanelt], cabbage (B. oleracea

Received for publication 20 Oct. 2008. Accepted for publication 10 Dec. 2008.

Funding for this work was partly provided by the 111 Project (no. B08025), the National 863 program (no. 2006AA10Z1C9) to X.L. Hou and the Key Project of Chinese Ministry of Education (no. 106092) to Y. Li. All are gratefully acknowledged. Several staff at the State Key Laboratory of Crop Genetics and Germplasm Enhancement, College of Horticulture, Nanjing, provided technical assistance. Genyi Li provided the sequences of SRAP primers. The anonymous reviewers provided critical comments and suggestions that helped improve the final version of the manuscript.

${ }^{1}$ Contributed equally to this paper.

${ }^{2}$ Corresponding author; e-mail hxl@njau.edu.cn.
L. var. capitata L.), broccoli (B. oleracea var. italica Plenck), cauliflower (B. oleracea var. botrytis L.), kale (B. oleracea var. viridis L.), and rape seed (B. rapa L. subsp. rapa) (Nozaki et al., 1997). Nonheading Chinese cabbage, which originated from China, is an important leafy vegetable in eastern Asia.

Many traits of agronomic importance are quantitative in nature and have complex genetic basis. The identification of quantitative trait loci (QTL) represents a first step toward dissecting the molecular basis of such complex traits. A prerequisite for QTL mapping studies is the availability of genetic maps, and many of these have been produced et al., 2007; Chyi et al., 1992; Kim et al., 2006; Nozaki et al., 1997; Soengas et al., 2007; Song et al., 1991; Suwabe et al., 2006; Teutonico and Osborn, 1994; Wu et al., 2008; Zhang et al., 2006). Geng et al. (2007) first for Brassica rapa (Ajisaka et al., 1995; Choi constructed a genetic linkage map of nonheading Chinese cabbage containing new, distorted, and missing markers using the method of Zhu et al. (2007). Knowledge of the location where these genes reside on the chromosomes would be useful to markerassisted selection, especially if easily measured molecular markers are closely linked to or associated with the specific QTL. In $B$. rapa, QTL analysis has been described for a wide variety of morphological and physiological traits such as seed color, pubescence, flowering time (Lou et al., 2007; Nozaki et al., 1995, 1997; Song et al., 1995; Teutonico and Osborn, 1995), oleic acid concentration (Tanhuanpaa et al., 1996), linolenic acid content (Tanhuanpaa and Schulman, 2002), clubroot resistance (Suwabe et al., 2006), black rot resistance (Soengas et al., 2007), and leaf mineral accumulation (Wu et al., 2008), but no information has been reported for nonheading Chinese cabbage.

Plant height is one of the important agronomic traits for plant breeding in nonheading Chinese cabbage and is affected by various environmental factors, including maturing, planting density, and climatic circumstances such as light, temperature, and water supply. QTL analysis for plant height in B. rapa has been carried out by several research groups (Lu et al., 2002; Song et al., 1995; Teutonico and Osborn, 1995; Yu et al., 2003). Usually, the phenotypic values for plant height at one specific developmental stage were used for QTL analysis. According to the rational points of developmental genetics, genes are expressed selectively at different growth stages. The conventional statistical results revealed that the development of morphological traits occurs through the actions and interactions of many genes that may behave differentially during growth periods and that gene expression is modified by interactions with other genes and by the environment (Atchley and Zhu, 1997). The genetic model from the final character cannot fully reflect the real gene action during the development of the character (Wu, 1987). It is necessary, therefore, to understand the dynamics of gene expression for a trait at different developmental stages as a basis for quantitative trait manipulation (Xu, 1997). In the last decade, molecular markers were applied to map QTL and to estimate their effects in different developmental stages (Bradshaw and Stettler, 1995; Plomion et al., 1996; Price and Tomos, 1997; Verhaegen et al., 1997). Zhu (1995) developed a genetic model to evaluate net genetic effects of quantitative traits at a specific developmental stage. This genetic effect is referred to as conditional genetic effect and the QTL that is detected at a specific growth interval with conditional genetic effect is referred to as conditional QTL. The phenotypic values of plant height are easy to score throughout the growth stage and have been used as model traits for time-dependent QTL mapping in rice (Cao et al., 2001; Yan et al., 1998), maize (Liu et al., 2007; Yan et al., 2003), and soybean (Sun et al., 2006). So far, no report has explored the developmental 
behavior of quantitative traits with molecular markers in Brassicas.

The objectives in this study were to add more molecular markers in the first genetic map of nonheading Chinese cabbage using the same doubled haploid (DH) population and to identify QTL for plant height using unconditional and conditional mapping method simultaneously in a series of measuring stages.

\section{Materials and Methods}

Plant materials. The mapping population, consisting of $112 \mathrm{DH}$ lines, was produced from a cross between two high inbred line parents, SW-13 and SU-124, through microspore culture of the $\mathrm{F}_{1}$ 'Shulv'. To produce a set of genetically uniform $F_{1}$ progeny, the cross was carried out by bud pollination with emasculation of SW-13 flowers. A genetic map with 138 markers was previously produced using this 'Shulv' DH population (Geng et al., 2007).

Construction of genetic linkage map. Leaf tissues were collected from the parents and 112 'Shulv' DH lines, immediately frozen, and later ground to powder in liquid nitrogen. Genomic DNA was extracted using the CTAB method (Murray and Thompson, 1998) with modification. The concentration of DNA was estimated using known concentration of lambda DNA in 1\% (w/v) agarose gel. The two parents were screened for polymorphism with sequence-related amplified polymorphism (SRAP), simple sequence repeat (SSR), random amplified polymorphic DNA (RAPD), and intersimple sequence repeat (ISSR) primer combinations (primers). The sequences of SRAP primers were provided by Dr. G. Li at the University of Manitoba, Canada. Primer sequences for SSR markers were obtained from Brassica microsatellite information exchange (http://www.brassica. info/ssr/SSRinfo.htm) and Cui et al. (2008). The sequence of RAPD and ISSR primers were obtained from Nanjing Sunshine Biotechology Co., Ltd. (Nanjing, China). The protocol of SRAP, RAPD, ISSR, and SSR markers assay was carried out as described by Geng et al. (2007). All these polymorphic markers between parents were tested against the DH line population. Genotypic data from the existing nonheading Chinese cabbage map with 77 SRAP markers, 27 SSR markers, 21 RAPD markers, and 13 ISSR markers were used together with the new additional SRAP, SSR, RAPD, and ISSR markers to construct a linkage map with software MAPMAKER 3.0/ EXP (Lander et al., 1987). Final map distances were calculated by applying the Kosambi function (Kosambi, 1944).

Field trials. Field trials were carried out over 2 years (2006 and 2007) from early September to middle December in Nanjing, China. All 112 DH lines, together with their parents and $F_{1}$, were grown in a randomized complete design with two replications. Thirty dry seeds were sown in a 10 -inch pot and seedlings were transferred to a higher field (20 cm higher than general field) $25 \mathrm{~d}$ later with a single plant spaced at $20 \times 25 \mathrm{~cm}$. Plant height was defined as the length from the surface of the soil to the tip of the plant. The day before transplanting, the first-stage data were measured from the central five plants (fixed throughout all measuring stages) from each plot. Ten days after transplanting, plant height was measured every $7 \mathrm{~d}$ until the middle of December. A total of eight different measurements were taken during the whole growth period of nonheading Chinese cabbage. Fertility and cultivation regimes were consistent with optimum production of nonheading Chinese cabbage for these regions.

Quantitative trait loci analysis. Phenotypic data for plant height in 'Shulv' DH population were analyzed by SPSS version 12.0.1 statistical package (SPSS Inc., Chicago, IL).

The analysis of unconditional and conditional QTLs was performed using QTL Cartographer with the composite interval mapping (CIM) module (Basten et al., 1996). Window size was $10 \mathrm{cM}$ and $5 \mathrm{cM}$, respectively. The threshold of QTLs score was identified by 1000 permutations using the Zmapqtl program in QTL Cartographer (Churchill and Doerge, 1994). A limit of detection (LOD) value corresponding to an experiment-wise threshold of $\alpha=0.05$ was used to declare a QTL as significant. The QTL position was estimated with the point of maximum LOD score in the region.

Unconditional QTL mapping was conducted based on phenotypic value at time $t[y(t)]$ (Zeng, 1993, 1994), in which the genetic effect was the accumulative of the individual gene effect from the initial time of plant growth to a time point $t$ :

$$
y_{j(t)}=\beta_{0(t)}+\beta_{(t)}^{*} X_{j}^{*}+\sum_{i} \beta_{(t)} X_{i j}+\varepsilon_{j(t)},
$$

where $y_{j(t)}$ is the phenotypic value of the $j$ th individual measured at time $t ; \beta_{0(t)}$ is the population mean at time $t ; \beta_{(t)}^{*}$ is the accumulated QTL effect at the time $t ; X_{(t)}^{*}$ is the coefficient for QTL effect; $\beta_{i(t)}$ is the accumulated effect for the $i$ th marker at the time $t ; X_{i j}$ is the coefficient for the $i$ th marker effect; and $\varepsilon_{j(t)}$ is the residual error of the $j$ th individual at the time $t$.

Conditional phenotypic mean $[y(t / t-1)]$, in which the genetic effect contributed by the specific developmental stage between time $t$-1 to time $t$ (Zeng, 1993, 1994):

$$
\begin{aligned}
y_{j(t \mid t-1)}= & \beta_{0(t \mid t-1)}+\beta_{(t \mid t-1)}^{*} X_{j}^{*} \\
& +\sum_{i} \beta_{(t \mid t-1)} X_{i j}+\varepsilon_{j(t \mid t-1)},
\end{aligned}
$$

where $y_{j(t \mid t-1)}$ is the conditional phenotypic value of the $j$ th individual; $\beta_{0(t \mid t-1)}$ is the conditional population mean; $\beta_{(t \mid t-1)}^{*}$ is the conditional QTL effect; $\beta_{i(t \mid t-1)}$ is conditional effect for the $i$ th marker; and $\varepsilon_{j(t \mid t-1)}$ is the conditional residual error of the $j$ th individual.

Conditional QTL analysis was conducted according to CIM along with the statistical method for the analysis of time-independent genetic effects (Zhu, 1995).

\section{Results}

Construction of genetic linkage map. As illustrated in Figure 1, the linkage map covered $1923.7 \mathrm{cM}$ of the genome of nonheading Chinese cabbage, and the average distance between markers was $10.3 \mathrm{cM}$ and the total number of markers to 186 . A variety of different types of molecular markers were used in the construction of the linkage map for the 'Shulv' DH population. Forty-eight newly genotyped markers (37 SRAPs, six SSRs, three RAPDs, two ISSRs) were integrated into Geng et al.'s (2007) previous map. The distribution of the individual marker types varied along the linkage groups (Fig. 1). The new genotyped markers located across 10 linkage groups ranged from three to 11 .

Phenotypic variation. The phenotypic values of plant height for 'Shulv' DH population, $F_{1}$, and their parents in eight measuring stages were collected in 2 years (Table 1). Compared with SW-13, parent SU-124 had always higher plants at all stages in 2 years. 'Shulv' DH population, $F_{1}$, and their parents had a slightly different plant height in 2007 and in 2006. $F_{1}$ showed obvious heterosis for plant height from the third stage. After the fifth stage, the growth of the plant became slower than former stages in 2 years, and it was the typical growth characteristics of the developmental trait (Wu et al., 2002). The plant height of the DH population segregated continuously and both skewness and kurtosis values were less than 1.0 in all measuring stages in 2006 and 2007. The data showed that the segregation of plant height fit a normal distribution for all growth stages. Transgressive segregants, with a plant height higher than parent SU-124 or lower than SW13 , were observed at all measuring stages in 2 years.

Unconditional quantitative trait loci for plant height development. The unconditional QTLs affecting plant height at different developmental stages in 2 years are presented in Table 2 and Figure 1. There were three, three, seven, three, three, four, five, and two unconditional QTLs detected in 2006 for each developmental stage, respectively, although two, one, three, one, one, one, five, and four unconditional QTLs were detected in 2007 for each developmental stage, respectively. The eight unconditional QTLs in 2006 were mapped on four linkage groups (LG2, LG3, LG4, and LG8), and the seven unconditional QTLs in 2007 were mapped on five linkage groups (LG2, LG3, LG8, LG11, and LG14). Maximum number of seven unconditional QTLs was found at the third stage in 2006 and five unconditional QTLs at the seventh stage in 2007. In 2006, two of eight unconditional QTLs (ph4 and ph8-1) were detected at only one developmental stage (seventh and third), whereas the other six unconditional QTLs (ph2-5, ph3-7, ph8-2, ph8-3, ph8-4, and ph8-5) were detected more than once at different measuring stages. In 2007, two of seven unconditional QTLs ( $p h 8$ 2 and ph14-2) were only detected at the 


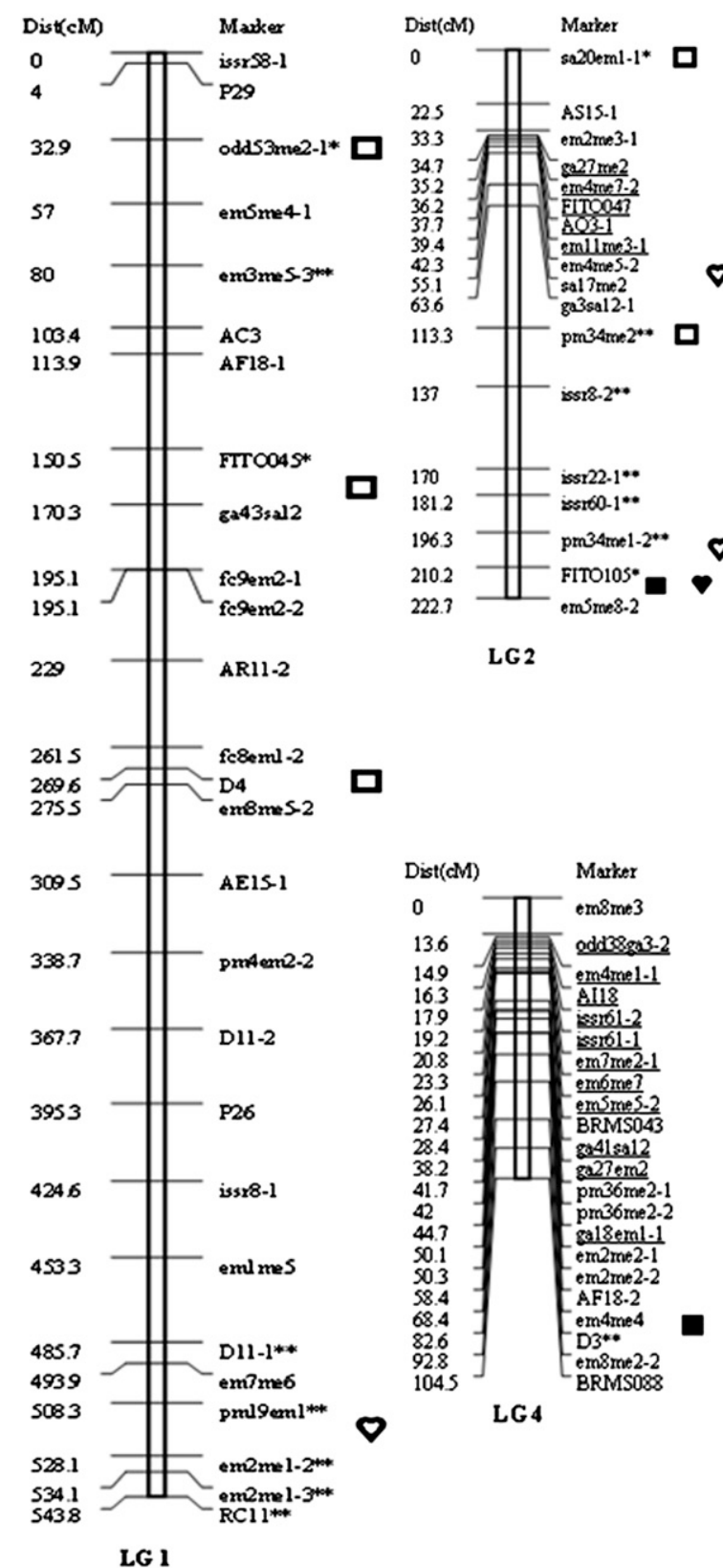

Fig. 1. (Continued on next page).

seventh stage, whereas the other five unconditional QTLs (ph2-5, ph3-4, ph8-4, ph8-5, and $p h 11)$ were detected more than once at different measuring stages. The contributions of these unconditional QTLs to phenotypic variance were $12.78 \%$ to $28.22 \%$ in 2006 and $7.92 \%$ to $28.25 \%$ in 2007 . The additive effects of these unconditional QTLs were from -1.87 to 1.33 in 2006 and from -1.96 to -0.72 in 2007 . There were four unconditional QTLs (ph2-5, ph8-2, ph8-4, and ph8-5) could be detected in both 2 years, although the significant stages showed some difference between the 2 years.

Conditional quantitative trait loci for plant height development. The analysis of conditional QTLs based on conditional phenotypic mean $\left[y_{j(t \mid t-1)}\right]$ was the result of the real temporal patterns of gene expression and provided inference for the net gene effects
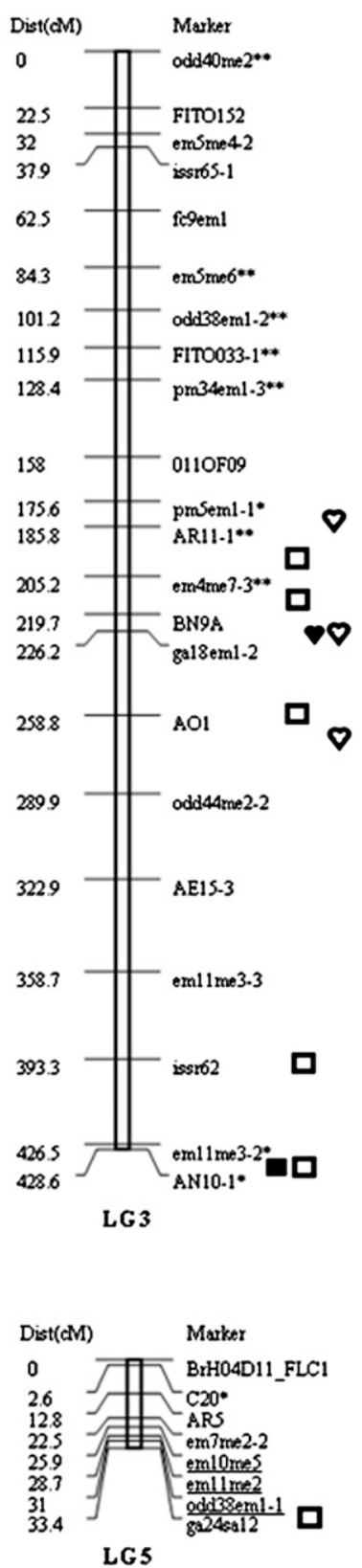

unconditional QTLs to phenotypic variance were from $8.05 \%$ to $24.33 \%$ in 2006 and from $7.92 \%$ to $19.21 \%$ in 2007 . The additive effects of these unconditional QTLs were from -1.29 to 0.49 in 2006 and from -1.16 to 0.49 in 2007 . There were five conditional QTLs (ph3-5, ph8-4, ph8-5, ph10, and ph14-1) could be identified in both years, although the significant stages showed some difference between the 2 years. All of the conditional QTLs in 2006 could be identified only at one specific stage, whereas two conditional QTLs (ph2-2 and ph2-4) could be identified at two different stages in 2007 .

\section{Discussion}

In the present study, the previous map (Geng et al., 2007) was saturated with 48 markers using the same DH population. This has resulted in an increase in the total number of markers to 186 . The map spans $1923.7 \mathrm{cM}$ and the markers are distributed over 14 linkage groups. Seven of these 14 linkage groups have more than 18 markers each, whereas the others contain only 10 or fewer markers. The average distance between the markers in our map is $10.3 \mathrm{cM}$, which is shorter than that reported in the previous map (Geng et al., 2007). However, the expected number of 10 linkage groups for a comprehensive linkage map of nonheading Chinese cabbage $(2 n=2 x=20)$ was exceeded by four linkage groups, of which one linkage group had only four markers. Because some linage groups are extremely small, it is safe to conclude that the apparent excess of linkage groups might be the result of incomplete coverage of the genome with the marker loci. To increase the map coverage, further research needs to be done through adding more individuals in the DH population, developing more molecular markers, and using different types of mapping populations such as $F_{2}$, recombinant inbred lines, and backcrosses.

According to the theory of developmental genetics, different genes express dynamically at different growth stages. Previous researches on QTL for plant height in $B$. rapa were focused on a single developmental stage, in which most of the genetic information was undetected. Song et al. (1995) mapped three QTLs for plant height in the $\mathrm{F}_{2}$ population of turnip rape and yellow sarson. Lu et al. (2002) mapped three QTLs for plant height in an $\mathrm{F}_{2}$ population of turnip and pakchoi. Yu et al. (2003) mapped six QTLs for plant height in RIL of Chinese cabbage. In this study, a total of 11 unconditional and 23 conditional QTLs for plant height were identified on nine linkage groups in 2 years. The numbers of QTLs related to plant height for nonheading Chinese cabbage as well as their genetic effects were varied at different measuring stages. Similar results have been observed in mapping QTLs for plant height of rice (Cao et al., 2001). This confirms the earlier statistical analysis results that gene action is distinct at various developmental stages, and genetic models of the final character cannot fully reflect the real 

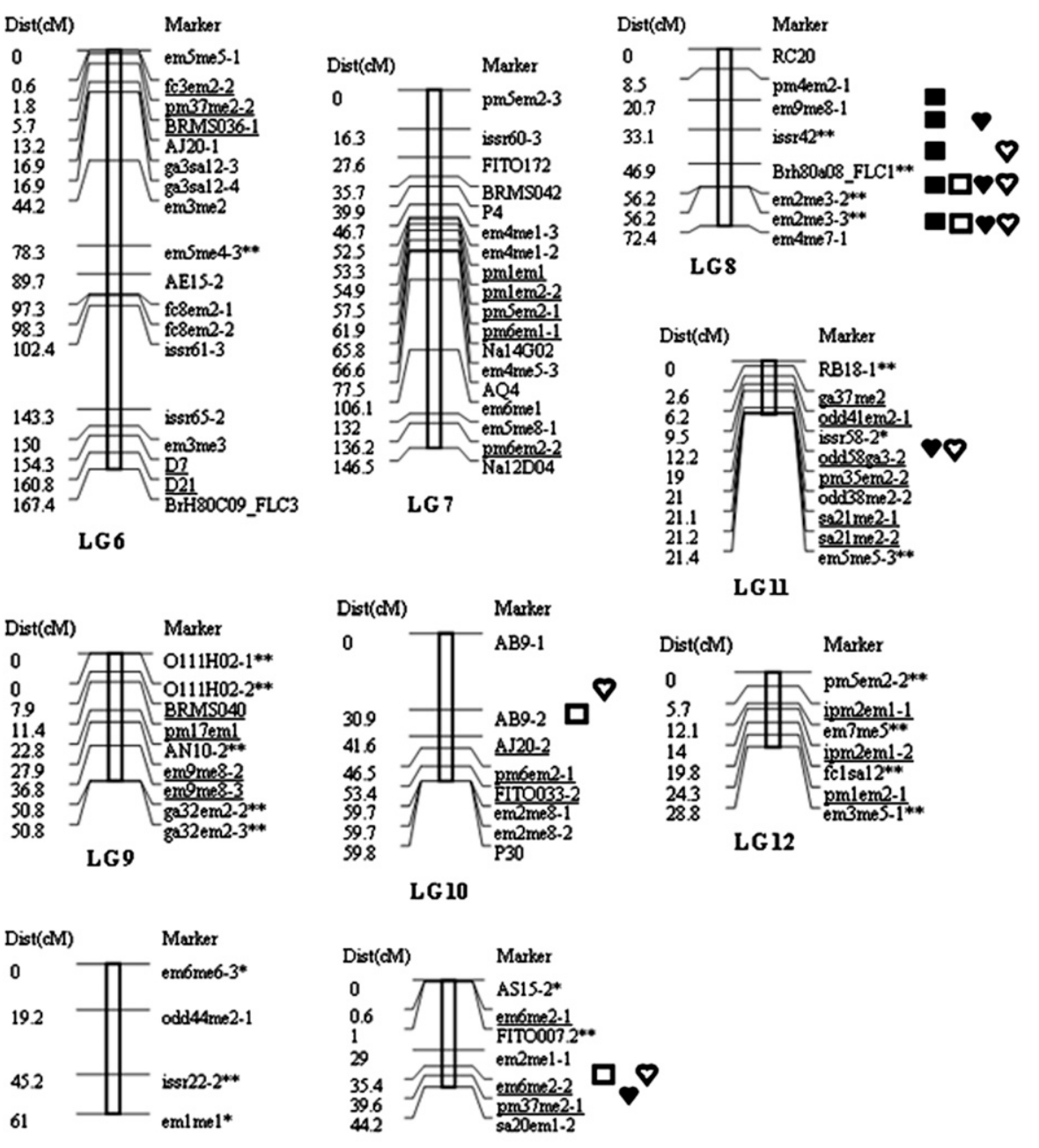

LG13

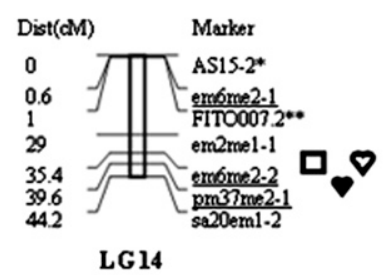

Fig. 1. Genetic linkage map of nonheading Chinese cabbage showing positions of unconditional and conditional quantitative trait loci for plant height at eight stages in 2 years. Mapped markers are indicated on the right and their corresponding genetic distances are indicated on the left in Kosambi centimorgans $(\mathrm{cM})$. Markers loci showing segregation distortion are indicated with asterisks; the number of asterisks indicates the level of significance with one symbol indicating significance at $P \leq$ 0.05 ; two at $P \leq 0.01$. New genotyped markers are underlined. Square symbols $=2006$; heart-shaped symbols $=2007$; closed symbols $=$ unconditional QTL; open symbols $=$ conditional QTL.

action of genes during the development of the character (Xu and Shen, 1991; Zhu, 1995).

Our results reveal that gene expression exhibited multiple patterns. Some gene effects maintain for a longer time and others disappear quickly. For instance, QTL ph2-2 was identified at two consistent stages of plant development in 2007, which could reflect a continuous expression of genes and QTL ph8-4, on the other hand, was identified at only one stage, which indicated gene expression at a specific measuring stage. Sun et al. (2006) made the similar conclusion for plant height in soybean. In contrast with our results, some QTLs developed for the number of rice tillers existed consistently for more than five measuring stages (Yan et al., 1998). The differences might be the result of the different nature for trait and background of genetic in monocot and dicot plants (Sun et al., 2006).

QTL $\times$ environment interaction is an important component affecting trait development, especially quantitative traits. QTLs detected explain the low percentage of the total phenotypical variance $(7.92 \%$ to $28.25 \%$ ), and this suggests the complex genetic control of plant height in this population. QTL detected in one environment but not in another environment may indicate a strong QTL $\times$ environment interaction (Veldboom and Lee, 1996a, 1996b).

Because negative additive effects meant the taller parent SU-124 provided the alleles to increase plant height, it is not surprising to find that all of the unconditional QTLs (except ph4 in 2006) and two-thirds of the conditional QTLs detected in 2 years had negative additive effects. However, we still found that the shorter parent SW-3 also provided the alleles to increase plant height. That means the alleles increasing plant height come from two parents synchronously (Xu, 1997) and reveals the genetic basis for the production of the transgressive segregation for plant height in the DH population. Furthermore, parental contribution of alleles at the same map position also changed along with the development of the trait. For example, conditional QTL ph2-4 with opposite genetic effects was detected for fifth and seventh stages in 2007. Similar gene expression patterns have also been observed in conditional QTL mapping for tiller number in rice (Yan et al., 1998).

Conditional QTL associated with plant height was mostly different from their unconditional QTL. In the present study, only four QTLs (ph3-4, ph3-7, ph8-4, and ph8-5)

Table 1. Phenotypic values of plant height $(\mathrm{cm})$ for the doubled haploid population, the hybrid, and their parents at eight different measuring stages in 2 years.

\begin{tabular}{|c|c|c|c|c|c|c|c|c|c|c|c|}
\hline \multirow[b]{2}{*}{ Stage } & \multirow[b]{2}{*}{ Year } & \multicolumn{2}{|c|}{ Parents } & \multirow{2}{*}{$\begin{array}{l}\text { Hybrid } \\
\text { Shulv }\end{array}$} & \multicolumn{7}{|c|}{ DH population } \\
\hline & & SW-13 & SU-124 & & Minimum & Maximum & Mean & SD & $\mathrm{CV}$ & Kurtosis & Skewness \\
\hline \multirow[t]{2}{*}{ First } & 2006 & 6.00 & 10.80 & 11.33 & 2.33 & 15.00 & 7.63 & 2.49 & 0.33 & 0.36 & 0.16 \\
\hline & 2007 & 6.23 & 11.17 & 11.67 & 2.13 & 15.27 & 7.64 & 2.56 & 0.34 & 0.31 & 0.18 \\
\hline \multirow[t]{2}{*}{ Second } & 2006 & 7.00 & 12.33 & 13.33 & 3.00 & 16.00 & 8.65 & 2.73 & 0.32 & 0.31 & 0.15 \\
\hline & 2007 & 6.87 & 12.23 & 13.67 & 3.33 & 16.37 & 11.43 & 2.86 & 0.31 & 0.33 & 0.21 \\
\hline \multirow[t]{2}{*}{ Third } & 2006 & 9.00 & 15.43 & 18.67 & 4.17 & 17.00 & 11.36 & 2.68 & 0.24 & -0.27 & -0.36 \\
\hline & 2007 & 8.87 & 15.33 & 19.23 & 4.33 & 17.17 & 11.43 & 2.94 & 0.21 & -0.22 & -0.31 \\
\hline \multirow[t]{2}{*}{ Fourth } & 2006 & 12.00 & 18.00 & 22.00 & 6.67 & 22.67 & 15.69 & 3.20 & 0.20 & -0.33 & -0.12 \\
\hline & 2007 & 12.13 & 17.97 & 22.13 & 6.67 & 23.33 & 16.17 & 3.12 & 0.22 & -0.31 & -0.18 \\
\hline \multirow[t]{2}{*}{ Fifth } & 2006 & 14.00 & 20.00 & 26.17 & 6.67 & 25.33 & 17.34 & 3.89 & 0.22 & -0.30 & -0.23 \\
\hline & 2007 & 14.33 & 20.15 & 26.66 & 7.17 & 25.67 & 17.37 & 3.65 & 0.23 & -0.29 & -0.25 \\
\hline \multirow[t]{2}{*}{ Sixth } & 2006 & 15.00 & 22.00 & 27.00 & 8.67 & 26.00 & 18.21 & 3.97 & 0.22 & -0.34 & -0.41 \\
\hline & 2007 & 15.32 & 21.96 & 27.26 & 8.67 & 26.27 & 18.23 & 3.99 & 0.20 & -0.31 & -0.39 \\
\hline \multirow[t]{2}{*}{ Seventh } & 2006 & 16.00 & 22.00 & 28.00 & 8.67 & 26.00 & 19.04 & 3.98 & 0.21 & -0.44 & -0.44 \\
\hline & 2007 & 15.99 & 22.21 & 28.22 & 9.00 & 26.67 & 19.43 & 3.89 & 0.22 & -0.39 & -0.48 \\
\hline \multirow[t]{2}{*}{ Eighth } & 2006 & 16.00 & 22.00 & 29.00 & 9.00 & 28.00 & 19.45 & 3.97 & 0.20 & -0.43 & -0.30 \\
\hline & 2007 & 16.21 & 22.32 & 30.11 & 9.33 & 28.13 & 19.54 & 4.10 & 0.21 & -0.41 & -0.36 \\
\hline
\end{tabular}


Table 2. Estimated unconditional and conditional genetic effects of quantitative trait loci (QTL) detected for the plant height of doubled haploid population at different stages based on composite interval mapping.

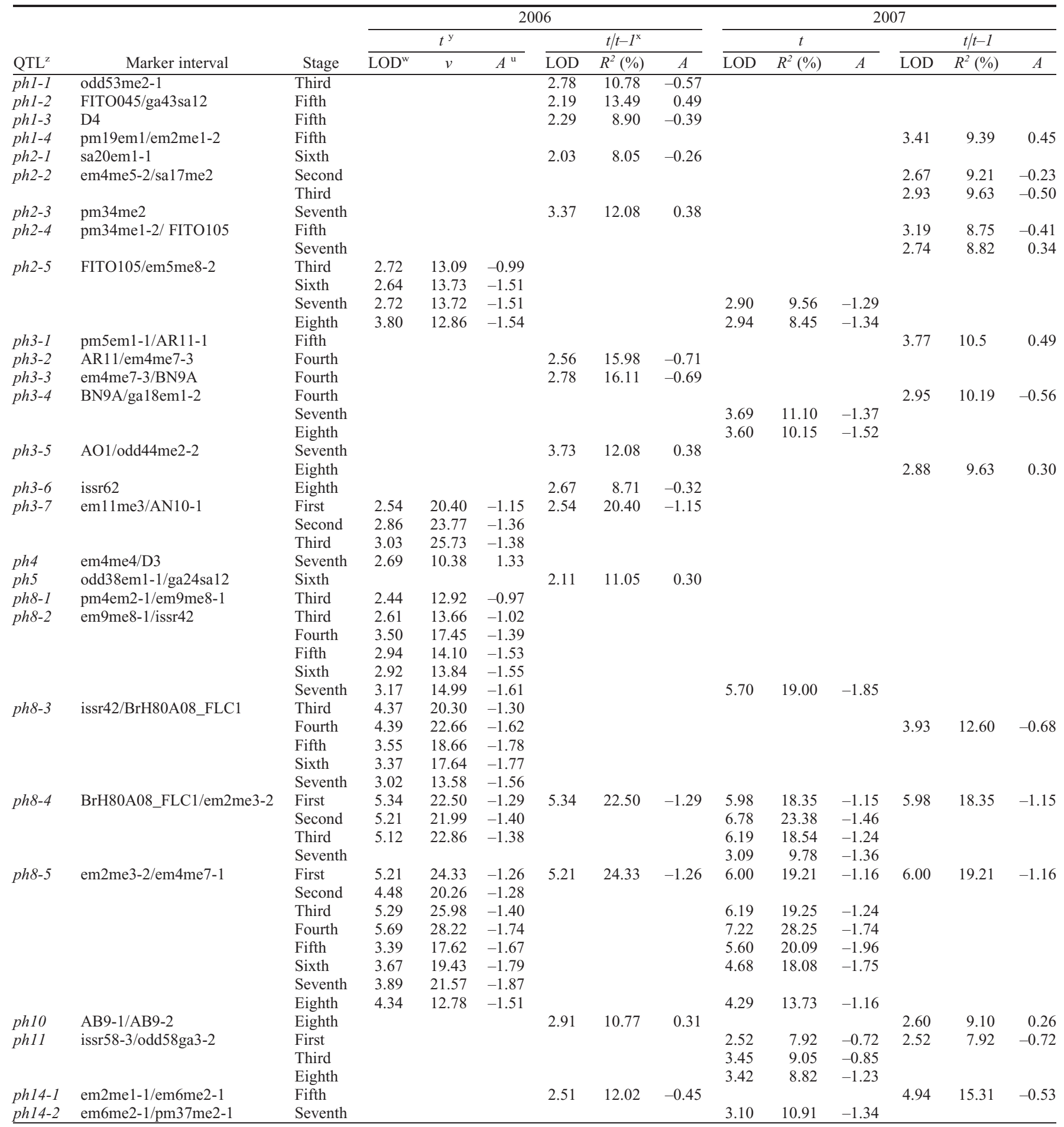

${ }^{\mathrm{z}}$ QTL names are abbreviations of the trait for plant height followed by its respective linkage group number. The serial number was added after the chromosomal number if more than one QTL was found in one linkage group.

${ }^{\mathrm{y}}$ Unconditional QTL.

${ }^{\mathrm{x}}$ Conditional QTL.

wMaximum-likelihood logarithm of the odds of linkage (LOD) score for the individual QTL.

${ }^{v}$ Proportion of the phenotypic variation explained by the QTL.

"Positive value implies that SW-13 has a positive effect on plant height and negative value implies that SU-124 has a positive effect on plant height.

detected were identical in both conditional and unconditional cases. Sun et al. (2006) reported a similar result for soybean pod number. The reason that the unconditional QTL is not be detected by the conditional method can be the minute expressions of the QTL at the multi- period. On the other hand, some QTLs undetected by the unconditional method are detected by the conditional method. This can be explained by the fact that the net effects of QTL from time $t-1$ to $t$ are partially counteracted by the accumulated effects before time $t-1$, and thus the QTL failed to be detected at time $t$ because the accumulated effects of them at this stage are too small. It is therefore suitable that the unconditional mapping method combined with the conditional mapping method is used in searching QTL. 
In our study, two QTLs (ph8-4 and ph8-5) were identified to be associated with plant height by unconditional and conditional mapping methods simultaneously in 2 years. These two QTLs could be the key genetic region (BrH80A08_FLC/em4me7-1) to control plant height in nonheading Chinese cabbage. However, the comparison of the present results with those reported previously in Chinese cabbage is difficult, because different markers and nomenclatures are used in different experiments. Although these regions need to be more precisely mapped, the information obtained should help in identification of candidate gene for plant height in nonheading Chinese cabbage.

\section{Literature Cited}

Ajisaka, H., Y. Kuginuki, K. Hida, S. Enomoto, and M. Hirai. 1995. A linkage map of DNA markers in Brassica campestris. Breed. Sci. 45(suppl):195-197.

Atchley, W.R. and J. Zhu. 1997. Developmental quantitative genetics, conditional epigenetic variability and growth in mice. Genetics 147:765-776.

Basten, C.J., B.S. Weir, and Z.B. Zeng. 1996. QTL Cartographer. North Carolina State University, Raleigh, NC.

Bradshaw, H.D. and R.F. Stettler. 1995. Molecular genetics of growth and development in Populus. IV. Mapping QTLs with large effects on growth, form, and phenology traits in a forest tree. Genetics 139:963-973.

Cao, G., J. Zhu, C. He, Y. Gao, J. Yan, and P. Wu. 2001. Impact of epistasis and QTL $\times$ environment interaction on the developmental behavior of plant height in rice (Oryza sativa L.). Theor. Appl. Genet. 103:153-160.

Choi, S.R., G.R. Teakle, P. Plaha, J.H. Kim, C.J. Allender, E. Beynon, Z.Y. Piao, P. Soengas, T.H. Han, G.J. King, G.C. Barker, P. Hand, D.J. Lydiate, D. Edwards, D.H. Koo, J.W. Bang, P. Beom-Seok, and Y.P. Lim. 2007. The reference genetic linkage map for the multinational Brassica rapa genome sequencing project. Theor. Appl. Genet. 115:777-792.

Churchill, R.W. and G.A. Doerge. 1994. Empirical threshold values for quantitative trait mapping. Genetics 138:963-971.

Chyi, Y.-S., M.E. Hoeneche, and J.L. Sernyk. 1992. A genetic linkage map of restriction fragment length polymorphism loci for Brassica rapa (syn. campestris). Genome 35:746-757.

Cui, X.M., Y.X. Dong, X.L. Hou, Y. Cheng, J.Y. Zhang, and M.F. Jin. 2008. Development and characterization of microsatellite markers in Brassica rapa ssp. chinensis and transferability among related species. Agr. Sci. China 7:257265.

Geng, J.F., C.S. Zhu, X.W. Zhang, Y. Cheng, Y.M. Zhang, and X.L. Hou. 2007. A genetic linkage map of nonheading Chinese cabbage. J. Amer. Soc. Hort. Sci. 132:816-823.

Kim, J.S., T.Y. Chung, G.J. King, M. Jin, T. Yang, Y. Jin, H. Kim, and B. Park. 2006. A sequencetagged linkage map of Brassica rapa. Genetics 174:29-39.

Kosambi, D.D. 1944. The estimation of map distance from recombination values. Ann. Eugen. 12:172-175.

Lander, E.S., P. Green, J. Abrahamson, A. Barlow, M.J. Daly, S.E. Lincoln, and L. Newburg. 1987. MAPMAKER: An interactive computer package for constructing primary genetic link- age maps of experimental and nature populations. Genomics 1:174-181.

Liu, Z.H., J.H. Tang, C.L. Wang, G.W. Tian, X.Y. Wei, Y.M. Hu, and D.Q. Cui. 2007. QTL analysis of plant height under N-stress and $\mathrm{N}$-input at different stages in Maize. Acta Agro. Sin. 33:782-789. [in Chinese].

Lou, P., J.J. Zhao, J.S. Kim, S.X. Shen, D.P. Del Carpio, X.F. Song, M. Jin, D. Vreugdenhil, X.W. Wang, M. Koornneef, and G. Bonnema. 2007. Quantitative trait loci for flowering time and morphological traits in multiple populations of Brassica rapa. J. Expt. Bot. 58:40054016.

Lu, G., J.S. Cao, H. Chen, and X. Xiang. 2002. QTLs mapping of some horticultural traits of Chinese cabbage. Sci. Agr. Sin. 35:969-974. [in Chinese].

Murray, M.G. and W.F. Thompson. 1998. Rapid isolation of high weight plant DNA. Nucleic Acids Res. 8:4231-4235.

Nozaki, T., M. Anji, T. Takahashi, and H. Ikehashi. 1995. Analysis of isozyme loci and their linkages in Brassica campestris L. Breed. Sci. 45:57-64.

Nozaki, T., A. Kumazaki, T. Koba, K. Ishikawa, and H. Ikehashi. 1997. Linkage analysis among loci for RAPDs, isozymes and some agronomic traits in Brassica campestris L. Euphytica 95: 115-123.

Plomion, C., C.E. Durel, and D.M. O'Malley. 1996. Genetic dissection of height in maritime pine seedlings raised under accelerated growth conditions. Theor. Appl. Genet. 93:849-858.

Price, A.H. and A.D. Tomos. 1997. Genetic dissection of root growth in rice (Oryza sativa L.). II. Mapping quantitative trait loci using molecular markers. Theor. Appl. Genet. 95:143-152.

Soengas, P., P. Hand, J.G. Vicente, J.M. Pole, and D.A.C. Pink. 2007. Identification of quantitative trait loci for resistance to Xanthomonas campestris pv. campestris in Brassica rapa. Theor. Appl. Genet. 114:637-645.

Song, K.M., M.K. Slocum, and T.C. Osborn. 1995 Molecular marker analysis of genes controlling morphological variation in Brassica rapa (syn. campestris). Theor. Appl. Genet. 90:1-10.

Song, K.M., J.Y. Suzuki, M.K. Slocum, P.H Williams, and T.C. Osborn. 1991. A linkage map of Brassica rapa (syn. campestris) based on restriction fragment length polymorphism loci. Theor. Appl. Genet. 82:296-304.

Sun, D.S., W.B. Li, Z.C. Zhang, Q.S. Chen, H.L. Ning, L.J. Qiu, and G.L. Sunday. 2006. Quantitative trait loci analysis for the developmental behavior of soybean (Glycine max L. Merr.). Theor. Appl. Genet. 112:665-673.

Suwabe, K., H. Tsukazaki, H. Iketani, K. Hatakeyama, M. Kondo, M. Fujimura, T. Nunome, H. Fukuoka, M. Hirai, and S. Matsumoto. 2006. Simple sequence repeat-based comparative genomics between Brassica rapa and Arabidopsis thaliana: The genetic origin of clubroot resistance. Genetics 173:309-319.

Tanhuanpaa, P.K. and A. Schulman. 2002. Mapping of genes affecting linolenic acid content in Brassica rapa ssp. oleifera. Mol. Breed. 10: 51-62.

Tanhuanpaa, P.K., J.P. Vilkki, and H.J. Vilkki. 1996. Mapping of a QTL for oleic acid concentration in spring turnip rape (Brassica rapa ssp. oleifera). Theor. Appl. Genet. 92:952-956.

Teutonico, R.A. and T.C. Osborn. 1994. Mapping of RFLP and quantitative loci in Brassica rapa and comparison to the linkage maps of $B$. napus, B. oleracea, and Arabidopsis thaliana. Theor. Appl. Genet. 89:885-894.
Teutonico, R.A. and T.C. Osborn. 1995. Mapping loci controlling vernalization requirement in Brassica rapa. Theor. Appl. Genet. 91:12791283.

Veldboom, L.R. and M. Lee. 1996a. Genetic mapping of quantitative trait loci in maize in stress and nonstress environments: I. Grain yield and yield components. Crop Sci. 36:13101319.

Veldboom, L.R. and M. Lee. 1996b. Genetic mapping of quantitative trait loci in maize in stress and nonstress environments: II. Grain yield and yield components. Crop Sci. 36: 1320-1327.

Verhaegen, D., C. Plomion, J.M. Gion, M. Poitel, P. Costa, and A. Kremer. 1997. Quantitative trait dissection analysis in Eucalyptus using RAPD markers. I. Detection of QTL in interspecific hybrid progeny, stability of QTL expression across different ages. Theor. Appl. Genet. 95:597-608.

Wu, J., Y.X. Yuan, X.W. Zhang, J.J. Zhao, X.F. Song, Y. Li, X.N. Li, R.F. Sun, M. Koornneef, M.G.M. Aarts, and X.W. Wang. 2008. Mapping QTLs for mineral accumulation and shoot dry biomass under different $\mathrm{Zn}$ nutritional conditions in Chinese cabbage (Brassica rapa L. ssp. pekinensis). Plant Soil 310:25-40.

$\mathrm{Wu}$, K.H. 1987. Analysis of gene effects for three quantitative characters at different development stages in maize. Acta Genet. Sin. 14:363-369.

Wu, W., Y. Zhou, W. Li, D. Mao, and Q. Chen. 2002. Mapping of quantitative trait loci based on growth models. Theor. Appl. Genet. 105:10431049.

Xu, Y.B. 1997. Quantitative trait loci: Separating, pyramiding, and cloning. Plant Breed. Rev. 15:85-139.

Xu, Y.B. and Z.T. Shen. 1991. Diallel analysis of tiller number at different growth stages for development behavior of tiller number in rice (Oryza sativa L.). Theor. Appl. Genet. 83:243249 .

Yan, J.B., H. Tang, Y.Q. Huang, Y.L. Zheng, and J.S. Li. 2003. Dynamic QTL analysis for plant height in different developing stages in maize. Chin. Sci. Bull. 48:1959-1964. [in Chinese]

Yan, J.Q., J. Zhu, C.X. He, M. Benmoussa, and P. Wu. 1998. Molecular dissection of developmental behavior of plant height in rice (Oryza sativa L.). Genetics 150:1257-1265.

Yu, S.C., Y.J. Wang, and X.Y. Zheng. 2003 Mapping and analysis QTL controlling some morphological traits in Chinese cabbage (Brassica campestris L. ssp. Pekinensis). Acta Genet. Sin. 30:1153-1160. [in Chinese].

Zeng, Z.B. 1993. Theoretical basis for separation of multiple linked gene effects in mapping quantitative trait loci. Proc. Natl. Acad. Sci. USA 90:10972-10976.

Zeng, Z.B. 1994. Precision mapping of quantitative trait loci. Genetics 140:745-754.

Zhang, X.W., J. Wu, J.J. Zhao, X.F. Song, Y. Li, Y.G. Zhang, D.H. Xu, R.F. Sun, Y.X. Yuan, C.H. Xie, and X.W. Wang. 2006. Identification of QTLs related to bolting in Brassica rapa ssp. pekinensis (syn. Brassica campestris ssp. pekinensis). Agr. Sci. China 5:265-271. [in Chinese].

Zhu, C.S., C.S. Wang, and Y.M. Zhang. 2007 Modeling segregation distortion for viability selection I. Reconstruction of genetic linkage maps with distorted markers. Theor. Appl. Genet. 114:295-305.

Zhu, J. 1995. Analysis of conditional genetic effects and variance components in developmental genetics. Genetics 141:1633-1639. 